\title{
Erythropoietin role in the therapeutic management of heart failure patients with anemia
}

Keywords: heart failure, angiotensin converting enzyme, ren inangiotensin, sympathetic systems, Sacubitril

Abbreviations: HF, heart failure; ACE, angiotensin converting enzyme; EPO, erythropoietin

\section{Editorial}

Heart failure (HF) is a serious public health problem of increasing magnitude and is the leading cause of hospital admission in developed countries. Despite the latest diagnostic and therapeutic advances its morbidity and mortality in the medium-term remains very high. ${ }^{1-3}$ The importance attributed to anemia in HF patients is increasing due to the relationship it has with its prognosis, which, despite all the treatments that have been used in HF continues to be poor. The data obtained confirms that anemia is clearly related to the poor prognosis of heart failure. ${ }^{4-6}$ This relationship had previously been observed by several authors, both in terms of mortality and the incidence of new hospital admissions for HF. Furthermore, this association has been observed

in hospitalized and outpatients. There is no doubt that correcting anemia can improve symptoms by correcting the oxygen supply to the tissues. Treatment with recombinant erythropoietin and parenteral iron improves the functional class, ventricular function, and quality of life of these patients, and also reduces the need for both oral and intravenous diuretics. $^{7-10}$

The introduction of beta adrenergic blockers associated with angiotensin converting enzyme (ACE) inhibitors in the treatment of HF led to a significant improvement in prognosis. ${ }^{11-13}$ The benefit obtained with beta-adrenergic blockers encouraged the search for new drugs that not only more completely block the activation of the reninangiotensin and sympathetic systems, but also allow modulating other activated phenomena in heart failure, such as inflammation and endothelial dysfunction. ${ }^{14}$ However, in recent years we have found that multiple studies analyzing the benefit of new drugs obtained controversial results. From new ACE inhibitors, endothelin and tumor necrosis factor alpha inhibitors, and the same angiotensin receptor antagonists that have brought benefits, but far below the expectations created. This indicates that there is probably no additional benefit to be gained by trying to increase the blockage of circulating neurohormones; in fact, some authors have suggested that this pathway has been exhausted and other therapeutic options must be sought. ${ }^{15}$ Current guidelines on heart failure recommend the use Sacubitril/ Valsartan $(\mathrm{S} / \mathrm{V})$, an angiotensin receptor- neprilysin inhibitor, in replacement of the renin-angiotensin-aldosterone system inhibition in ambulatory patients with HF and reduced ejection fraction still symptomatic despite optimal medical therapy. ${ }^{16}$ This recommendation comes from a single randomized study named PARADIGM-HF trial, which showed the superiority of Sacubitril-Valsartan compared to Enalapril in reducing the incidence of cardiovascular death or hospitalizations for HF. ${ }^{17}$ Nevertheless, despite the improvements in clinical management and medical therapy of HF, the outcome of these patients still remains poor. ${ }^{18}$
Volume 13 Issue 3 - 2020

\author{
Osmar Antonio Centurión, 1,2 Juan D \\ Cáceres' \\ 'Department of Health Sciences Investigation, Sanatorio \\ Metropolitano, Paraguay \\ ${ }^{2}$ Cardiology Department, Clinical Hospital, Asunción National \\ University (UNA), Paraguay
}

Correspondence: Osmar Antonio Centurión, Professor of Medicine, Asuncion National University, Department of Health Sciences Investigation, Sanatorio Metropolitano, Teniente Ettiene $215 \mathrm{c} /$ Ruta Mariscal Estigarribia Fernando de la Mora, Paraguay, Tel +595-97I-354444, Emailosmarcenturion@hotmail.com

Received: May 27, 2020 | Published: June 17, 2020

The persistence of significant ventricular remodeling despite optimized medical treatment has been associated with a poorer prognosis in heart failure. In this sense, possible interventions to cut the signals that activate the mechanisms that mediate progressive ventricular remodeling are being investigated in recent years. Despite the effort made, there is still a long way to go before it can become a reality that allows newer drugs to be generated. ${ }^{19}$ The lack of effective new treatments has led to a deeper analysis of the factors that affect the prognosis of heart failure, and anemia is one of them. Increasing attention is paid to anemia in patients with HF due to the relationship it has with its prognosis, which, despite all the treatments that have been used in HF, continues to be poor. ${ }^{5,6}$ This relationship had previously been observed by several authors, both in terms of mortality and the need for new hospital admissions for HF. Furthermore, this association has been observed in hospitalized and outpatients. In fact, anemia is usually frequent in patients with HF in advanced stages of the disease. There is no doubt that correcting anemia can improve symptoms by correcting the oxygen supply to the tissues. Treatment with recombinant erythropoietin (EPO) and parenteral iron improves the functional class, ventricular function, and quality of life of these patients and also reduces the need for diuretics, both oral and intravenous..$^{7-10}$

The prevalence of anemia in HF is highly variable and can range from 5 to $55 \%$, depending on the series. This wide variability is due to the different criteria used for its diagnosis and the differences in the populations studied which may lead to disparities in clinical results. ${ }^{4}$ The presence of anemia is usually higher in epidemiological studies with older patients, a worse functional class of the New York Heart Association and more comorbidity. While in pharmacological studies, where patients tend to be younger and more selected the prevalence of anemia decreases markedly. ${ }^{1,20}$ One of the factors that greatly increase the prevalence of anemia is the presence of chronic renal failure which is often multifactorial in HF. The stipulated prevalence was 
$30 \%$ for a population of 64 years of average age, with a predominance of men, few cases with associated renal failure and in the control in tertiary hospitals. It is possible that in older patients, with a higher incidence of women and associated kidney failure, the prevalence is much higher. ${ }^{21-23}$

In recent years, numerous studies have linked a decrease in hemoglobin with an increase in mortality. The fact that the correction of anemia can promote new treatment routes is still controversial. In this sense, it must be borne in mind that anemia in HF can be secondary to very diverse causes. ${ }^{4-6}$ Thus, at the onset of the disease it is unlikely to have significant prognostic implications and in some cases it may even be secondary to hidden losses in patients on aspirin treatment. In fact, in series with relatively young patients referred for heart transplantation, the presence of anemia was not a determining factor in overall mortality. ${ }^{24}$

As heart failure progresses, much comorbidity are associated with it that may promote anemia. A retrospective study found that anemia was not due only to heart failure, but to other causes in $98 \%$ of cases, the most frequent of which was associated chronic kidney failure, followed by iron, folic acid deficiency or vitamin B12. ${ }^{5}$ What does seem certain is that in the advanced phase of the disease there are multiple mechanisms that can cause anemia. The activation of cytokines may favor cachexia, chronic malnutrition, hemodilution, and worsening kidney failure as it reduces cardiac output, less perfusion of the bone marrow that would reduce its regenerative response. However, it is possible that in many cases the etiology of HF anemia is related to the release of cytokines with the ability to inhibit erythropoiesis characteristic of chronic diseases and is closely related to the age of the patients and their functional status. ${ }^{6}$ It is striking that in many series the cause of anemia was not studied in more than half of the cases, reflecting the low relevance that clinicians give it in the context of an elderly patient with a poor baseline situation. In fact all these mechanisms can be found simultaneously in terminal HF and interact with each other, making it very difficult to establish the ultimate cause of anemia and assess its true prognostic implication.

Observational studies indicated that treatment with EPO might reduce HF hospitalizations and improve exercise capacity and quality of life through correction of anaemia. ${ }^{25,26}$ Indeed, patients treated with an EPO showed a decreased risk of hospitalization for HF in an interesting meta-analysis. ${ }^{27}$ Adverse events reported in relation to 2 different moments of drug application should be considered: in initial uncontrolled clinical trials in which adverse events are reported and the accumulated experience showing that most of them are not a direct cause of EPO.$^{27}$ High blood pressure appears as the only adverse event demonstrated and associated with this, migraine headache in children; therefore, severe hypertension must be controlled prior to the start of treatment. Associated hypertensive encephalopathy is less and less frequent. Other possible adverse events not demonstrated over time are: seizures, thrombosis of the vascular access. No worsening of renal function has been established in the pre-dialysis stage. ${ }^{27}$

A study conducted in Israel evaluated circulating serum erythropoietin levels as a prognosis for patients with HF in 188 outpatients. ${ }^{28}$ The authors prospectively evaluated the predictive value of erythropoietin, N-terminal pro-B natriuretic peptide, and C-Reactive Protein in patients with clinically stable chronic HF. Erythropoietin levels were found to be directly related to the New York Heart Association functional class of HF. ${ }^{28}$ They conclude that longer-term studies are needed for determination of circulating levels of erythropoietin to assist in predicting morbidity and mortality in patients with clinically controlled heart failure. ${ }^{28}$ Swedberg K et al. ${ }^{29}$ studied in a randomized, double-blind trial, 2278 patients with systolic heart failure and mild-to- moderate anemia (hemoglobin level, 9.0 to $12.0 \mathrm{~g}$ per deciliter). They were to receive either darbepoetin alfa (to achieve a hemoglobin target of $13 \mathrm{~g}$ per deciliter) or placebo. The primary outcome was a composite of death from any cause or hospitalization for worsening heart failure. The authors reported that primary outcome occurred in 576 of 1136 patients $(50.7 \%)$ in the darbepoetin alfa group and 565 of 1142 patients (49.5\%) in the placebo group. There was no significant between-group difference in any of the secondary outcomes. The neutral effect of darbepoetin alfa was consistent across all pre-specified subgroups. Fatal or nonfatal stroke occurred in 42 patients $(3.7 \%)$ in the darbepoetin alfa group and 31 patients $(2.7 \%)$ in the placebo group $(\mathrm{P}=0.23)$. Thromboembolic adverse events were reported in 153 patients $(13.5 \%)$ in the darbepoetin alfa group and 114 patients $(10.0 \%)$ in the placebo group $(\mathrm{P}=0.01)$. Cancer-related adverse events were similar in the two study groups. Therefore, the authors ${ }^{29}$ concluded that treatment with darbepoetin alfa did not improve clinical outcomes in patients with systolic heart failure and mild-to-moderate anemia. There was a significant increase in the risk of thromboembolic events among patients receiving darbepoetin alfa. Our findings do not support the use of darbepoetin alfa in these patients. The findings of Swedberg K et al. ${ }^{29}$ suggest that the hemoglobin level, like other surrogates, is simply a marker of poor prognosis in heart failure rather than a therapeutic target. However, an important limitation of their study was the exclusion of patients with severe anemia (hemoglobin level, $<9.0 \mathrm{~g} / \mathrm{dL}$ ). Such patients might have a greater increase in hemoglobin values with darbepoetin alfa, thereby greater separation from the findings in the placebo group.

On the other hand, although the rate of clinical events was not reduced in the darbepoetin alfa group, treatment of anemia did improve the quality of life assessed by the Overall Summary and Symptom Frequency Scores on the KCCQ, as evidenced by a 2.2 point higher KCCQ score compared with the placebo group $(\mathrm{P}=0.005)$. This improvement in quality of life was probably due to the fact that Darbepoetin alfa treatment led to an early and sustained significant increase of $1.8 \mathrm{~g} / \mathrm{dL}$ in the hemoglobin level. In the treatment group, median hemoglobin levels increased from to $13 \mathrm{~g} / \mathrm{dL} .^{29}$ Further subgroup analysis revealed that patients requiring only a low dose EPO to restore their hemoglobin values may have benefited from EPO treatment, as their bone marrow was more responsive. More support for low dose EPO comes from studies exploring extra-hematopoeitic effects, namely, the capacity to induce neovascularization. ${ }^{28}$ As capillary density in HF patients is decreased, these patients may still benefit from low dose EPO. Therefore, further studies are needed to see if there is still a niche for EPO in certain HF patients with severe anemia.

\section{Acknowledgments}

None.

\section{Conflicts of interest}

The authors declare there are no conflicts of interest related to the article.

\section{Funding}

None. 


\section{References}

1. Ponikowski P, Voors AA, Anker SD, et al. 2016 ESC Guidelines for the Diagnosis and Treatment of Acute and Chronic Heart Failure. Rev Esp Cardiol. 2016;69(12):1167.

2. Yancy CW, Jessup M, Bozkurt B, et al. 2013 ACCF/AHA guideline for the management of heart failure: executive summary: a report of the American College of Cardiology Foundation/American Heart Association Task Force on practice guidelines. Circulation. 2013;128(16):1810-1852.

3. Martindale JL. Wakai A, Collins SP, et al. Diagnosing Acute Heart Failure in the Emergency Department: A Systematic Review and Meta-analysis. Academic Emergency Medicine. 2016;23(3):223-242.

4. O'Meara E, Murphy C, McMurray JJ. Anemia and heart failure. Curr Heart Fail Rep. 2004;1:176-182.

5. Anand I, McMurray JJ, Whitmore J, et al. Anemia and its relationship to clinical outcome in heart failure. Circulation. 2004;110(2):149-154.

6. Maggioni AP, Opasich C, Anand I, et al. Anemia in patients with heart failure: prevalence and prognostic role in a controlled trial and in clinical practice. J Card Fail. 2005;11(2):91-98.

7. Cleland JG, Sullivan JT, Ball S, et al. Once-monthly administration of darbepoetin alfa for the treatment of patients with chronic heart failure and anemia: a pharmacokinetic and pharmacodynamics investigation. $J$ Cardiovasc Pharmacol. 2005;46:155-161.

8. Ngo K, Kotecha D, Walters JA, et al. Erythropoiesis-stimulating agents for anemia in chronic heart failure patients. Cochrane Database Syst Rev. 2010;1:CD007613.

9. Ghali JK, Anand IS, Abraham WT, et al. Randomized double-blind trial of darbepoetin alfa in patients with symptomatic heart failure and anemia. Circulation. 2008;117(4):526-535.

10. Ponikowski P, Anker SD, Szachniewicz J, et al. Effect of darbepoetin alfa on exercise tolerance in anemic patients with symptomatic chronic heart failure: a randomized, double-blind, placebo-controlled trial. $J$ Am Coll Cardiol. 2007;49(7):753-762.

11. Tai C, Gan T, Zou L, et al. Effect of angiotensin-converting enzyme inhibitors and angiotensin II receptor blockers on cardiovascular events in patients with heart failure: a meta-analysis of randomized controlled trials. BMC Cardiovasc Disord. 2017;17(1):257.

12. Martin N, Manoharan K, Thomas J, et al. Beta-blockers and inhibitors of the renin-angiotensin aldosterone system for chronic heart failure with preserved ejection fraction. Cochrane Database Syst Rev. 2018;6(6):CD012721.

13. Turgeon RD, Kolber MR, Loewen P, et al. Higher versus lower doses of ACE inhibitors, angiotensin-2 receptor blockers and beta- blockers in heart failure with reduced ejection fraction: Systematic review and metaanalysis. PLoS One. 2019;14(2):e0212907.

14. George J, Patal S, Wexler D, et al. Circulating erythropoietin levels and prognosis in patients with congestive heart failure: comparison with neurohormonal and inflammatory markers. Arch Intern Med. 2005;165(11):1304-1309.

15. Kuster GM, Pfister O. Chronic heart failure: advances in pharmacological treatment and future perspectives. Swiss Med Wkly. 2019;149:w20036.
16. Ponikowski P, Voors AA, Anker SD, et al. ESC Guidelines for the diagnosis and treatment of acute and chronic heart failure: The Task Force for the diagnosis and treatment of acute and chronic heart failure of the European Society of Cardiology (ESC). Developed with the special contribution of the Heart Failure Association (HFA) of the ESC. Eur J Heart Fail. 2016;18(8):891-975.

17. McMurray JJ, Packer M, Desai AS, et al. Angiotensin-neprilysin inhibition versus enalapril in heart failure. N Engl J Med. 2014;371(11):993-1004.

18. Jhund PS, Macintyre K, Simpson CR, et al. Long-term trends in first hospitalization for heart failure and subsequent survival between 1986 and 2003: a population study of 5.1 million people. Circulation. 2009;119(4)515-523.

19. Castro-Torres Y, Katholi RE. Recently approved and under investigation drugs for treating patients with heart failure. Curr Cardiol Rev. 2020.

20. McMurray JJ, Adamopoulos S, Anker SD, et al. ESC Committee for Practice Guidelines. ESC guidelines for the diagnosis and treatment of acute and chronic heart failure 2012: the Task Force for the Diagnosis and Treatment of Acute and Chronic Heart Failure 2012 of the European Society of Cardiology. Developed in collaboration with the Heart Failure Association (HFA) of the ESC. Eur J Heart Fail. 2012;14:803-809.

21. Pfeffer MA, Burdmann EA, Chen CY, et al. A trial of darbepoetin alfa in type 2 diabetes and chronic kidney disease. $N$ Engl $J$ Med. 2009;361:2019-2032.

22. Singh AK, Szczech L, Tang KL, et al. Correction of anemia with epoetin alfa in chronic kidney disease. N Engl J Med 2006;355:2085-2098.

23. Besarab A, Bolton WK, Browne JK, et al. The effects of normal as compared with low hematocrit values in patients with cardiac disease who are receiving hemodialysis and epoetin. N Engl J Med. 1998;339(9):584590.

24. Ezekowitz J., McAlister FA, Armstrong PW, et al. Anemia Is Common in Heart Failure and Is Associated With Poor Outcomes. Insights from a Cohort of 12065 Patients With New-Onset Heart Failure. Circulation. 2003;107(2):223

25. van Veldhuisen DJ, Dickstein K, Cohen-Solal A, et al. Randomized, double- blind, placebo controlled study to evaluate the effect of two dosing regimens of darbepoetin alfa in patients with heart failure and anaemia. Eur Heart J. 2007;28(18):2208-2216.

26. Ghali JK, Anand IS, Abraham WT, et al. Study of Anemia in Heart Failure Trial (STAMINA-HeFT) Group. Randomized double blind trial of darbepoetin alfa in patients with symptomatic heart failure and anemia. Circulation. 2008;117(4):526-535.

27. van der Meer P, Groenveld HF, Januzzi JL Jr, et al. Erythropoietin treatment in patients with chronic heart failure: a meta-analysis. Heart. 2009;95:1309-1314.

28. Manolis AS, Tzeis S, Triantafyllou K, et al. Erythropoietin in heart failure and other cardiovascular diseases: hematopoietic and pleiotropic effects. Curr Drug Targets Cardiovasc Haematol Disord. 2005;5(5):355-375.

29. Swedberg K, Young JB, Anand IS, et al. Treatment of Anemia with Darbepoetin Alfa in Systolic Heart Failure. N Engl J Med. 2013;368:12101219. 